\title{
Need of Herbal Antibiotics
}

\section{Priti $\mathbf{M}^{*}$}

Department of Biotechnology, Amity University, India

Editorial

Volume 2 Issue 1

Received Date: July 20, 2018

Published Date: July 26, 2018

DOI: $10.23880 /$ cprj-16000110

Institute of Biotechnology, Amity University, Uttar Pradesh, Mallhore, Lucknow-226028,

India, Tel: +91-9695961829; Email: preitymathur@gmail.com

\section{Editorial}

During the last two decades, the development of drug resistance as well as the appearance of undesirable side effects of certain antibiotics has led to the search of new therapeutic agents. The problem of antibiotic resistance is so alarming that the World Health Organization (WHO) has also warned that we may possibly enter a "postantibiotic era", when even very common infections will no longer have a treatment. Currently, there are large numbers of problems with the treatment of diseases caused by clinically important bacterial strains that display multidrug-resistance (MDR) property. Today, approximately $30 \%$ of the infections are due to vancomycin resistant Enterococci, while approximately $60 \%$ of infections are due to methicillin-resistant $S$. aureus. There is no perfect treatment for tuberculosis.Currently even after the improvements in health care system \& the accessibility of a sufficient number of medicines, death rates are still high due to infections with resistant. The occurrence of bacterial resistance is not new, it was first identified by Sir A. Fleming with respect to penicillin more than 50 years ago. This problem has emerged as a serious public health concern as the effectiveness of more \& more antibiotics is reduced.It has been expected that, if no new antibiotics are recognized by 2050, millions of people will die globally each year as a direct consequence of bacterial resistant infections. These problems have emerged as serious public health concerns as the effectiveness of antibiotics are reduced. It has been expected that, if no new antibiotics are recognized by 2050, millions of people will die globally each year as a direct consequence of bacterial resistant infections. This increasing problem has forced many researchers to search for new effective antibacterial therapies from natural sources that would combat resistant pathogens.

Dependence on plants as a source of medication is common in developing nations where traditional treatment plays an important role in primary health care. According to WHO report, nearly $80 \%$ of individual from these countries still use plants as remedies from many diseases, using their own personal recipes which have been pass through generations. The use of plants as a source of medication is common in developing nations where traditional treatment plays an important role in primary health care .According to WHO report, nearly $80 \%$ of individual from these countries still use plants as remedies from many diseases, using their own personal recipes which have been pass through generations. Additionally, the National Health Interview Survey (NHIS) directed by the Centres for Disease Control (CDC) specify that nearly $40 \%$ of adults in the United States used certain form of alternative therapies, suggesting that such health practices are predominantly performed even in the developed countries .It has been predicted that there are 3,00000 to 5,00000 different plant species in the world with biologically \& chemically diverse groups. According to one report approximately 2,00000 Secondary metabolisms present as specialized compounds.The existence of this biologically active substance within the plant tissue is responsible for its therapeutic value \& includes alkaloids, glycosides, flavonoids, tannins, resins, essential oils, gums, etc. These phytochemicals provide a frequent motivation of therapeutic agent with reduced toxicity, broad spectrum of activity \& good pharmacokinetics to be used clinically deprived of any chemical alteration, they also have the ability to modify 


\section{Clinical Pathology \& Research Journal}

the resistance associated with MDR strains\& hence, has been the topic of interest since long time.There have been a large amount of phytochemicals associated with antibacterial efficacy; they are still not used as commercial antibiotics so far. Therefore, plants must be explored to better recognize their therapeutic values, safety \& efficacy, so that they can replace \& used as an alternate of synthetic drugs. 